\title{
Clasificación de las expresiones eidéticas. El caso de los «biosferismos»
}

Christian Álvarez-Rojas ${ }^{1}$

D) 0000-0001-9800-1834

\section{Resumen}

En el siguiente trabajo presento una clasificación de las expresiones eidéticas, reformulando los criterios expuestos por Eduardo Devés y Andrés Kozel en Estudios Eidéticos. Propongo dos ejes, uno de complejidad y alcance, y otro genealógico-ontogenético, los cuales describen variables sincrónicas y diacrónicas, respectivamente. Finalmente, propondré a modo de ejemplo analítico al conjunto de los «biosferismos», entendido como ideas sobre el ambiente que requieren previamente una concepción planetaria y científica de la vida, aun cuando sus intereses específicos sean locales o con objetivos preferentemente éticos o políticos

\section{Abstract}

In the following paper I present a classification of eidetic expressions, reformulating the criteria set forth by Eduardo Devés and Andrés Kozel in Estudios Eidéticos. I propose two axes, one of complexity and scope, and another of genealogy-ontogeny, which describe synchronic and diachronic variables, respectively. Finally, I will propose as an analytical example the set of "biospherisms", understood as ideas about the environment that require a planetary and scientific conception of life, even when their specific interests are local or with preferably ethical or political objectives.

1 Dr. (C) en Estudios Americanos, especialidad Pensamiento y Cultura. Instituto de Estudios Avanzados, Universidad de Santiago de Chile. 


\section{La clasificación de las ideas}

Un aspecto indispensable del estudio de cualquier rincón de la realidad es la clasificación. Desde asumir, al menos en términos prácticos y metodológicos, que un tipo de fenómenos es distinto a los otros, hasta descubrir o proponer diferencias internas en esta distinción. En los estudios de las ideas, la clasificación misma constituye un paso adelante en la producción del conocimiento, desde la «ideología» de Destutt de Tracy y las continuas reformulaciones de este concepto hechas por Marx, Gramsci o Althusser.

En la propuesta presentada en Estudios Eidéticos, Eduardo Devés y Andrés Kozel establecen la distinción necesaria entre los tipos de entidades eidéticas, partiendo de la constatación de que, por ejemplo, decir «la ideología de Jesús» o «la cosmovisión postestructuralista» resulta contraproducente tanto por anacronismo como por la falta de límites mínimos en el alcance de los conceptos. Es así como se plantean distintos criterios de clasificación, los cuales operan de forma simultánea y no aspiran a formar un todo orgánico o jerárquico. Lo más cercano a ello, sin embargo, es la clasificación que, para efectos de este trabajo, reformulo como de extensión. En este criterio las expresiones eidéticas se pueden clasificar en cosmovisiones, teologías, sistemas filosóficos, ideologías, paradigmas científicos, dispositivos eidéticos, cotidianías, hasta llegar a una unidad hipotética mínima, al estilo del atomismo de Demócrito, de eidas, es decir, de ideas como «lucha de clases» o «todas las personas nacen libres e iguales» que, al modo de los aminoácidos, se encadenan formando el código geneidético de los distintos tipos de expresiones eidéticas, con la posibilidad de mezclarse de forma ilimitada para dar origen a cruces 
como «socialismo islámico» o «cientificismo espiritual» (Álvarez 2020).

Otras clasificaciones propuestas en Estudios Eidéticos son:

Según código geneidético (familia): Es posible establecer genealogías y parentescos entre expresiones eidéticas según las eidas compartidas, permitiendo clasificaciones linneanas y/o darwinianas. Por ejemplo, la eida «el estado como instrumento al servicio de la burguesía» forma parte del socialismo y también del anarquismo, los cuales, a su vez, comparten el rechazo al tradicionalismo del Antiguo Régimen, emparentándose más lejanamente con el liberalismo y el positivismo en tanto ideologías y filosofías propias de la Ilustración.

Según función: identitaria / centralitaria, disciplinadora / de rebelión, cosmopolita / nacionalista, cohesiva / confrontativa, expansionista / rechazo o expulsión del invasor. Un ejemplo es el nacionalismo, un conjunto de ideas que, como en el caso del eslavismo durante el siglo XIX, tuvo funciones cohesivas y de rebelión, mientras que en el siglo XXI el eslavismo promovido por la política exterior rusa, reivindicando el pensamiento de Ivan Ilyn, adquiere funciones disciplinadoras y expansionistas (Snyder 2018).

Según grado de complejidad (cantidad de ideas): Es la premisa metodológica que considera, por ejemplo, a las cosmovisiones como portadoras de más eidas que las cotidianías, a los sistemas filosóficos como más complejos que las ideologías.

Según grado de agencia: cuasinulo, bajo, alto, muy alto (Devés y Kozel 2017, p. 329-31).

Como propuesta de trabajo, propongo reformular estas clasificaciones siguiendo dos ejes principales: a) complejidad y alcance $\mathrm{y}$ b) 
ontogénesis genealogía. En el criterio de complejidad y alcance, asumo la posibilidad de clasificar las expresiones eidéticas en un espectro que va de un grado menor a mayor, donde la complejidad va aparejada con el nivel explicativo que se espera de las ideas, es decir, si se refieren a asuntos concretos, ligados a problemas específicos, o si sirven como explicaciones para áreas más extensas de la realidad, como la polis, la vida como fenómeno natural, o incluso la realidad completa. En este punto, soy enfático en señalar que asumimos a lo que las ideas proponen, sin considerar su potencial validez epistemológica (como al considerar a la cosmogonía helénica como «explicación del origen del mundo») y, sobre todo, que dada la naturaleza del objeto de estudio, esta clasificación es un supuesto metodológico, sin pretender asignarle un compromiso ontológico fuerte que niegue la posibilidad de otras clasificaciones complementarias, o la existencia de ideas que permitan un solapamiento de las categorías.

Eje de complejidad y alcance:

Eidas $<$ Dispositivo eidético (nacionalismo, cientificismo) $<$ Cotidianías (futbolismo, jardinerismo, ver Devés 2021a) < Ideologías (liberalismo, marxismo) < Teologías, Sistemas filosóficos, paradigmas científicos < ideoglobías (Devés 2021b) < cosmovisiones (kyme mogen mapuche, ver Loncon Antileo 2017, cosmogonía helénica)

En el eje ontogénesis-genealogía, asumo que las expresiones eidéticas aparecen históricamente como variaciones de expresiones anteriores, asumiendo una creciente diferenciación y especificidad en la medida en que aumenta la eidodiversidad. Toda expresión eidética contemporánea podría, en principio, ser rastreada según sus componentes hacia expresiones anteriores que la influyeron, hasta 
llegar a las cosmovisiones que, en tiempos pre-civilizatorios, fueron la expresión eidética predominante en nuestros ancestros.

Una formulación de esta genealogía tentativa sería: cosmovisiones > teologías $>$ sistemas filosóficos $>$ paradigmas científicos $>$ ideologías $>$ ideoglobías $>$ cotidianías. A diferencia de la ontogénesis biológica, los tipos de ideas no mueren, sino que disminuyen su alcance o permanecen en estado de latencia, más allá de que expresiones puntuales desaparezcan sin haber dejado rastros, como la cosmovisión de los habitantes del asentamiento de Monte Verde. Esta clasificación se presenta como un modelo de análisis, asumiendo las limitaciones de este método, como describe Grace Lindsay: «all models are wrong, because all models ignore some details. All models are also wrong because they represent only a biased view of the processes they claim to capture. And all models are wrong because they favour simplicity over absolute accuracy. All models are wrong the same way all poems are wrong; they capture an essence, if not a perfect literal truth» (2021, p. 15).

\section{Los biosferismos}

En esta sección presentaré, mediante ejemplos, la categoría de expresiones eidéticas «biosferismos», aludiendo a aquellas ideas que, en una génesis contemporánea, y con un alcance local en comparación a las ideologías o las cosmologías, contienen como núcleo el cuidado del ambiente como condición necesaria para la supervivencia humana. Refiero al concepto «biósfera» porque remite a la constatación empírica, y ya no solo a la deducción racional, de que habitamos un planeta finito, cuyos recursos, desde la propia superficie a la energía y 
los alimentos, exigen que adoptemos una normatividad que limite nuestras conductas de consumo. En este punto, se implica también la constatación de la existencia de una economía capitalista global, de un Sistema-Mundo del que se forma parte en sus centros o periferias.

El concepto biosferismo ya ha sido utilizado en la psicología social como rasgo de personalidad que no es individualista y que manifiesta la prosocialidad como cuidado del ambiente. Este perfil psicológico logra predecir conductas y disposiciones individuales en torno a valores altruistas, considerando a la naturaleza como un valor en sí mismo antes que con consideraciones utilitaristas (Stern 2000; Gatersleben et al 2014; de Groot \& Steg 2008; Amérigo \& Bernardo 2007).

Desde los estudios de las ideas, y refiriendo a la constatación que propongo, un antecedente importante está en La metafísica de las costumbres de Kant, donde la finitud de la superficie terrestre, constatada con precisión e incluida dentro del sentido común tras la Era de los Descubrimientos, motiva una necesidad primigenia de ordenar las voluntades humanas para buscar una coexistencia pacífica: «puesto que la tierra no es ilimitada sino que es una superficie limitada por sí misma, [se conduce] inevitablemente a la idea de un derecho político de gentes (ius gentiun) o un derecho cosmopolita (ius cosmopoliticum)»(140).

La finitud como variable permanente fue un aspecto central en el pensamiento económico clásico, especialmente en Malthus, Ricardo y Marx, sin embargo, la posibilidad de avances técnicos que solucionaran la escasez de recursos naturales, por la superación de la dependencia respecto a estos, impidió que ideas biosferistas tuvieran una posición central. Debemos esperar hasta el siglo XX para que esto ocurra, con la constatación de la extrema degradación ambiental, ya no por polución 
atmosférica o deforestación, únicamente, sino por un conjunto multifactorial que incluye la sobreexplotación y contaminación de los océanos, la reducción de la biodiversidad, y el impacto de residuos industriales, llegando a alterar la formación de los tejidos humanos en el embarazo (Ragusa et al 2021).

Una expresión eidética relevante, que aparece como síntoma del creciente escepticismo sobre la posibilidad de desacoplar el crecimiento económico del uso de recursos naturales esencialmente finitos, lo constituye el concepto Spaceship Earth, que alude tanto al carácter de finitud ineludible como de la exigencia normativa de lograr una administración justa y sostenible de los recursos, como si el planeta fuese una nave de la que como humanidad somos la tripulación. Este enfoque adquirió notoriedad en la obra de economistas e ingenieros como Barbara Ward (1966), Kenneth Boulding (2017 [1966]) y Buckminster Fuller (1978), siendo un insumo conceptual clave para el informe sobre los límites del crecimiento, en el cual Donella Meadows y el Club de Roma establecen la conclusión, cada vez más resonante, de que no es posible un crecimiento infinito en un planeta de recursos finitos (Meadows \& Club of Rome 1972).

Esta constatación, sin embargo, no está exenta de usos ideológicos que, de forma explícita y explícita, contribuyen a mantener el propio capitalismo global que causa la devastación ecológica denunciada. En su estudio sobre el ambientalismo como producto de la Guerra Fría, Perrin Selcer plantea que: «[t]he biosphere frame focused attention on the imperative to analyze cause- and- effect relationships at the world scale and to use science to guide the managerial state. The metaphor of Spaceship Earth fused this technocratic vision with the ideals of world community. In 1972, the UN Conference on the Human 
Environment (UNCHE) in Stockholm sought to establish the principles» (2018, p. 174, énfasis nuestro). La protección del ambiente como «fin en sí mismo» no está ajena a los intereses políticos y económicos de los estados que las administran, incluyendo las potenciales influencias epistémicas de estos criterios en principio extracientíficos. En su estudio crítico sobre la metáfora de la Tierra como nave, Sabine Höller plantea:

[T]he figure of Spaceship Earth was not simply a discursive invention that could be altered at will, but rather a material-semiotic intervention, an unprecedented realization [...] The conservative attitude of nurture and protection towards a nature wild, pure, pastoral, sublime and even divine, kept within distinct boundaries, followed national, territorial and often utilitarian ideals. A hundred years later, nature was conceived of not in immediate national and recreational terms but in the functional terms of a global ecosystem from which humankind profited in the mediated ways of systems dynamics. The 'biosphere reserves' of the early 1970s emerged out of global initiative; they were based on international networks and on different epistemological frameworks: although as exclusionary as a park or reserve, they expressed a new form of nature preservation on a scientific basis, in which selected areas were protected according to their representative ecosystem functions. $(2015$, p. 13)

Los impactos de la constatación de la imposibilidad de un crecimiento ilimitado en la geopolítica mundial fueron advertidos tempranamente desde el pensamiento latinoamericano. La perspectiva del Club de Roma fue recogida en la convocatoria de la primera Conferencia de las Naciones Unidas sobre el Medio Ambiente Humano realizada en Estocolmo en 1972, donde los países del Primer Mundo, si bien «utilizaron brillantemente una retórica que planteaba que la crisis 
ambiental amenazaba al conjunto de la humanidad, lo que realmente los motivaba era la amenaza que podía significar, para sus sociedades hegemónicas y dominantes, el agravamiento de una crisis que ellos mismos habían generado con su proceso de crecimiento y desarrollo, si las sociedades periféricas intentaban copiar su "éxito"» (Estenssoro, p. 49).

Un modelo que contestó el enfoque del Norte fue el propuesto en el informe ¿Catástrofe o Nueva Sociedad? Modelo Mundial Latinoamericano de la Fundación Bariloche, publicado en 1978 bajo la dirección de Amílcar Herrera. En él, se plantea que el deterioro ambiental no es «una consecuencia inevitable del progreso humano, sino el resultado de una organización social cimentada en valores gran parte destructivos», cuya solución o agravamiento no depende «en última instancia de barreras físicas insuperables, sino de factores sociales y políticos que a los hombres compete modificar» (cit. en Estenssoro, p. 59). Otra crítica relevante es la del economista brasileño Ignacy Sachs, quien formula el concepto de ecodesarrollo en oposición a la posición del Club de Roma y del Norte Global en Estocolmo 72, considerando la mención a los límites físicos «una banalidad» (p. 62), siendo lo verdaderamente relevante la utilización de la ciencia y la tecnología para mejorar la calidad de vida de la población sin una lógica de acumulación capitalista.

A partir de esta trayectoria conceptual, los biosferismos son ideas que orientan acciones concretas sobre la degradación inviable de la naturaleza, considerando que esta es un resultado del proceso de expansión global del capitalismo (lo que lleva, en potencia, a buscar alternativas a este) y, sobre todo, en un plano local, particular, tal vez como aceptación de la complejidad estructural de la crisis. 
Un ejemplo de biosferismo es el ecofeminismo, que aúna reivindicaciones de género con críticas sistémicas a nivel económico y tecnológico, ofreciendo soluciones locales. La filósofa india Vandana Shiva «critica el modelo económico dominante, ya que propaga las técnicas de plantación de monocultivos tanto en los bosques como en la agricultura; considera que el sistema económico indio tradicional preserva la relación mutua con la naturaleza a través del policultivo, cuyo objetivo es la producción de subsistencia local con insumos propios», además «ha extendido este análisis a todos los sectores del aparato productivo capitalista, condenando sus sistemas tecnológicos y organización del trabajo» (Carcaño Valencia, p. 184). La socióloga argentina Maristela Svampa plantea de forma más directa que «[n]o hay feminismo emancipador si no lleva en el centro de su mensaje la defensa del territorio y la vida. Ser feminista y no ser ecologista es casi una contradicción hoy en día» (cit. en Figueroa 2020). Este vínculo se da por convergencia en el proceso que la autora llama «feminización de las luchas», en el cual las mujeres han devenido en figuras clave en las demandas colectivas: «en nuestros trabajos previos de investigación, hemos constatado que muy poca gente se reconoce inicialmente como "ambientalista" o "ecologista". Tampoco se deviene ambientalista por elección, sino por obligación, a partir de la asunción de la lucha por la defensa de la vida y el territorio» (Svampa, 2015, p. 128). La lucha territorial implica, además, prácticas comunitarias vernáculas que deben aprovecharse: «en el contexto de las actuales resistencias al extractivismo, el lenguaje de valoración de las mujeres enmarcado en la cultura del cuidado tiende a expresar un ethos procomunal potencialmente radical, que concibe las relaciones sociales desde otra 
lógica y otra racionalidad, cuestionando el hecho capitalista desde el reconocimiento de la ecodependencia y la valoración del trabajo de reproducción de lo social» (p. 131).

El indigenismo, entendido como un conjunto pluralista de pensamiento contemporáneo desarrollado por pueblos indígenas, también forma parta de los biosferismos. Un ejemplo de pensamiento indigenista, el Sumak Kawsay, se puede considerar un biosferismo en tanto:

Seus proponentes procuraram demonstrar suas possibilidades em ao menos três direções: na construção de relações globais menos desiguais, mais cooperativas e horizontalizadas; numa abordagem mais holística das questões ambientais, integrando humanidade e natureza e formulando novos direitos humanos, como à água e a um ambiente são; e em propostas em torno da defesa dos povos indígenas, em contextos de experimentação de formas de estados plurinacionais e de pluralismo jurídico. (Pereira da Silva, p. 185)

Es decir, el rescate conceptual de prácticas culturales ancestrales no corresponde a una visión idealizada del pasado al que se debe retornar, sino a la recuperación de un acervo de ideas que permita pensar alternativas a las crisis ambientales y económicas del presente. Fabricio Pereira da Silva constata, además, que «[g]eralmente, seus defensores propõem superar o extrativismo que constitui a base das economias primárias daqueles países» (Ibíd.), con lo que se incluye en el diagnóstico la exclusión del Sur Global de las decisiones sobre el ambiente tomadas en la institucionalidad del Norte, ya mencionado anteriormente.

En su intervención en el Foro Permanente para las Cuestiones Indígenas de las Naciones Unidas del 2008, Evo Morales expuso los 
Diez Mandamientos para salvar al planeta, a la humanidad y a la vida inspirados en el Sumak Kawsay, destacando en ellos la contingencia producida por el capitalismo global y la escasez de recursos:

Los Diez Mandamientos son: Primero: Terminar, acabar con, erradicar el sistema capitalista Segundo: Renunciar a la guerra Tercero: Un mundo sin imperialismo ni colonialismo. Cuarto: El agua es de todos los seres vivientes Quinto: Desarrollo de energías limpias y amigables con la naturaleza Sexto: Respeto a la Madre Tierra Séptimo: Servicios básicos como derecho humano Octavo: Consumir lo necesario, priorizar lo que producimos y consumimos localmente Noveno: Promover la diversidad de culturas y economías Décimo: El Vivir Bien. (Ministerio de Relaciones Exteriores, 2010, p. 16, énfasis nuestro)

Si sumamos, además, que los estados de Ecuador y Bolivia han propiciado que el pluralismo jurídico de estos conceptos ofrezca un nuevo marco de relaciones internacionales, tenemos una dimensión biosferista que no estaba presente, al menos con este énfasis, en el pensamiento de autores clásicos como Fausto Reinaga o José Carlos Mariátegui.

La apelación a particularidades culturales como herramienta jurídica para impedir el deterioro ambiental, si bien ha sido rechazada desde la institucionalidad internacional (Estenssoro, 2019) ha tenido resultados concretos a nivel local. Un ejemplo es la victoria judicial de comunidades diaguita en el norte de Chile que obligó a paralizar la actividad minera en el Glaciar Pascua Lama, hito que fue comunicado de la siguiente forma por sus representantes: 
Este cierre significa avanzar hacia la liberación de nuestro territorio sagrado de empresas multinacionales y nacionales que lo destruyen, además, de ser una posibilidad para que nuestros glaciares -o bancos perpetuos como le denominaban nuestros abuelos- sigan vivos $\mathrm{y}$ surtan de agua de manera permanente al río, que es el espíritu de este valle. (OCMAL)

Estos éxitos, aunque momentáneos, orientan a los biosferismos a acciones acotadas tanto en lo temático como en lo geográfico, sin que pierdan de su marco general de análisis el diagnóstico global de la crisis que enfrentan. Como último ejemplo de expresión eidética biosferista, me referiré al veganismo, entendido no solo como el abandono del consumo de productos derivados de la explotación animal, sino además como un desarrollo teórico que busca convencer de que esta posición es indispensable para combatir el impacto ambiental del capitalismo. En su exposición sobre el veganismo, la bióloga brasileña Paula Brügger plantea que:

O veganismo é um poderoso vetor de mudança rumo a uma ética ecocêntrica. E a dieta vegana, em escala planetária, promoveria o resgate e a manutenção das diversidades gen(éticas) de uma forma muito mais eficiente do que quaisquer atividades e projetos que hoje visam a essa finalidade, além de contemplar outras dimensões imprescindíveis para se alcançar a sustentabilidade. (2009, p. 208)

En la ética biocéntrica mencionada confluyen razonamientos tanto deontológicos, propios del biosferismo como rasgo psicológico, de valorar a la naturaleza como «bien en sí mismo», y también utilitaristas, en donde se asume que «[s]er estritamente vegano é quase impossível, visto que nossa sociedade usa derivados de animais nos mais diversos setores produtivos» (p. 206), pero sí es posible y deseable avanzar en la reducción gradual del consumo, así como aminorar el 
sufrimiento de los animales no humanos. En su introducción al veganismo, Patti Nyman enfatiza en la estabilidad de la propia alimentación humana de mantenerse la división vigente entre el consumo y producción Norte-Sur:

Una cuarta parte de todo el grano producido en los países del tercer mundo se da al ganado. Esto afecta los precios y la disponibilidad de los alimentos, y la seguridad alimentaria. El crecimiento de la población humana en la actualidad está creando cada día 230 mil nuevas bocas que alimentar. Qué tipo de alimentos se cultivan y cuál es la eficiencia de este crecimiento en términos de uso de la tierra y los recursos determinará si podemos mantener la vida humana en la tierra [...] Estamos produciendo comida más que suficiente para alimentar a la población humana, pero en cambio estamos alimentando los animales, agotando los recursos y destruyendo el planeta en el proceso. (p. 24-5)

Constatamos, entonces, que el veganismo como expresión eidética, si bien inicia su reflexión a partir del cuidado ético de los animales no humanos, se distancia de pensamientos con vocación similar, como el jainismo, al incluir el consumo de productos animales en un sistema global de explotación de potencial insostenible, ya no, únicamente, por premisas éticas, sino apelando a la mejor evidencia científica disponible. 


\section{Conclusiones y precauciones}

Cada expresión eidética mencionada como una forma de biosferismo constituye en sí misma una familia amplia de propuestas a ser examinada en detalle, y en las cuales pueden existir premisas potencialmente contradictorias o indiferentes a las variables consideradas fundamentales para un pensamiento biosferista. Tal posibilidad constituye, a mi juicio, un resultado esperable de ideas emergidas en un momento de alta circulación de ideas entre distintos espacios geográficos, con una participación inédita de voces enfrentadas a un escenario global común. Entiendo a la disonancia cognitiva como un componente ineludible del razonamiento humano (Sperber \& Hirschfeld, 1999), cuya superación solo es posible con la especialización excepcional de la modelación científica o la sistematización filosófica. Lo relevante es identificar cómo, ante un escenario de crisis ambiental, se plantean propuestas que la visualizan como un problema científico, político y económico global, al mismo tiempo que gestionando intervenciones particulares en lo temático y geográfico.

Los biosferismos expuestos en este trabajo comparten, además de las variables definitorias, el ser ideas con fines emancipatorios, que cuestionan un sistema político-económico global para volverlo «más justo», o bien reemplazarlo por un sistema nuevo que sí cumpla con las promesas ilustradas de igualdad, libertad y fraternidad. Este rasgo, sin embargo, es circunstancial, puede estar perfectamente ausente en otras variedades de biosferismos, como ocurre con el ecofascismo. Conviene enfatizar esta variedad del fascismo respecto a sus expresiones históricas anteriores, pues si bien el nazismo tuvo un componente «ecologista» al promover el cuidado de su «espacio vital», también 
propuso un desarrollo industrial ineludible y que debía ser orientado por las ciencias biológicas (Zimmerman, 1995, p. 212). El fascismo contemporáneo, además, presenta vertientes abiertamente antiecologistas, en donde la destrucción ambiental se considera un insumo político en favor de instalar un gobierno autoritario (Alizart, 2020).

El ecofascismo bebe de la ecología profunda, expresión eidética iniciada por el filósofo noruego Arne Naess, la cual se podría redefinir como el paso consciente del biosferismo como rasgo psicológico a una posición normativa en términos éticos y políticos, a partir de la arbitrariedad del antropocentrismo y la constatación del lugar noprivilegiado de nuestra especie en la biósfera. Aún cuando Naess fue abiertamente antifascista, participando en la resistencia armada a la ocupación nazi de Noruega, basado en su convencimiento de una dignidad humana universal (van Boeckel, 1995), su pensamiento ha sido continuamente reivindicado por ecofascistas, especialmente por los alcances demográficos de la ecología profunda. Al respecto, Arne Naess sostuvo que: «[t]he flourishing of human life and cultures is compatible with a substantial decrease of the human population. The flourishing of nonhuman life requires such a decrease» (1992, p, 29).

En su análisis del pensamiento del ecofascista finés Kaarlo Pentti Linkola, Evangelos Protopapadakis constata que el rechazo a la supremacía del Homo sapiens conlleva a una vocación restauradora que avanza el diagnóstico de Naess a la acción: «[i]f any particular species interacts with its environment in a way that is detrimental to the ecosphere, the situation needs to be altered in such a way so as to allow the reestablishment of proper equilibrium-even if this would call for the destruction of the harmful species» (2014, p. 589). Linkola, en 
tanto ecofascista prototípico, no objeta ningún medio que reduzca la población humana con tal de que se reduzca su impacto ambiental: «[a]ny means to decreasing human population would be welcomed with relief [...]; even war, genocide, and disease, as long as any of these would be massively destructive for the species Homo sapiens» (p. 5912).

Estas ideas ya han sido reivindicadas en actos terroristas, como el ocurrido el 15 de marzo de 2019 en Christchurch, Nueva Zelanda, en donde antes de asesinar a 51 personas y herir a otras 40 en una mezquita, su autor publicó un manifiesto en donde se proclamó «ecofascista» y afirmó que «no hay nacionalismo sin ambientalismo» (Stern, 2019). El 3 de agosto del mismo año, en Texas, otro terrorista asesinó a 23 personas e hirió al mismo número por tratarse de «latinos» que, en tanto «invasores», serían responsables de la degradación ambiental y el agotamiento de los recursos, según lo publicado en su manifiesto; "[i]f we can get rid of enough people, then our way of life can be more sustainable» (Nast, 2019).

Dada la crisis climática en curso y en aumento, así como la escasez de recursos, ya vislumbrada hace décadas por las potencias globales, resulta crucial considerar a los biosferismos como ideas que tendrán un rol movilizador creciente, no solo a nivel de activismo, sino también en términos ideológicos (cómo administrar la polis) y disciplinares, con objetivos tan opuestos como cumplir al fin los ideales ilustrados o los reaccionarios. La administración utilitaria de la demografía, propia del ecofascismo, y evidente en su expresión más grotesca en las reivindicaciones terroristas, está presente de forma más sutil en las planificaciones estratégicas de las potencias frente al cambio climático 
y la escasez de recursos como «amenaza para la seguridad», en donde la posición hegemónica de los estados del Norte Global, con sus respectivos niveles de gasto energético, se asume invariable, quedando como única alternativa el control de las regiones periféricas. En su revisión de los planes de EE. UU. y la Unión Europea para enfrentar militarmente el aumento de las migraciones desde el Sur Global, Nick Buxton observa que: «those who are the least responsible for climate change are not only the most affected by it, but are also viewed as "threats"» (2021, p. 9).

Los estudios eidéticos, como programa de investigación, tienen entonces una tarea ineludible: exponer que las ideas, aun cuando apunten a la resolución de problemas urgentes y con un sentido de justicia, pueden seguir vinculadas a posturas éticas que querríamos creer ya enterradas en el suelo histórico. Suelo fértil que permite la latencia y el retorno, los que son una constante y que, ocasionalmente, como en el presente, constituyen no solo un interés académico sino una amenaza terminal. 


\section{Bibliografía}

Alizart, M. (2020). Golpe de estado climático. La Cebra.

Álvarez-Rojas, C. (2020). Cientificismo espiritual: La obra de Arturo Aldunate Philips en la discusión sobre ciencia y desarrollo. Límite, 15. https://doi.org/10.4067/S0718-50652020000100204

Amérigo, M., \& Bernardo, A. (2007). Representación social del ser humano versus naturaleza y su relación con las creencias medioambientales. Revista de Psicología Social, 22(3), 219-233. https://doi.org/10.1174/021347407782194399

Boulding, K. E. (2017). The economics of the coming spaceship earth. En A. Markandya \& J. A. Richardson (Eds.), The Earthscan reader in environmental economics (pp. 27-35). Earthscan Publications.

Brügger, P. (2009). Nós e os outros animais: Especismo, veganismo e educação ambiental. Linhas Críticas, 15(29), 197-214.

Buxton, N. (2021). A primer on climate security The dangers of militarising the climate crisis. Transnational Institute.

Carcaño Valencia, É. (2008). Ecofeminismo y ambientalismo feminista: Una reflexión crítica. Argumentos (México, D.F.), 21, 183-188.

http://www.scielo.org.mx/scielo.php?script=sci_arttext\&pid=S0187$\underline{57952008000100010 \& \mathrm{nrm}=\text { iso }}$

de Groot, J. I. M., \& Steg, L. (2008). Value Orientations to Explain Beliefs Related to Environmental Significant Behavior: How to Measure Egoistic, Altruistic, and Biospheric Value Orientations. Environment and Behavior, 40(3), 330-354. https://doi.org/10.1177/0013916506297831

Observatorio de Conflictos Mineros de América Latina. (2020) Declaración de comunidades diaguitas ante el cierre Pascua Lama. 2020, octubre 14. https://www.ocmal.org/declaracion-de-comunidades-diaguitas-ante-el-cierrepascua-lama/

Devés, E. (2021a). “Cotidianías" en América Latina actual: Conceptos, géneros, contrastes. Estudios Avanzados, 34, 1-14.

https://doi.org/10.35588/estudav.v0i34.4824

(2021b). ¿Una nueva especie de ideas? Las “ideoglobías” como propuestas para una mejor convivencia global en la época contemporánea: concepto y casos. En-Claves del pensamiento. https://doi.org/10.46530/ecdp.v0i30.461

Devés, E., \& Kozel, A. (2018). Estudios eidéticos. Una conversación desde el Sur sobre la vida de las ideas y la reconfiguración de un espacio disciplinar. Teseo. 
Estenssoro, F. (2019). La geopolítica ambiental global del siglo XXI: Los desafíos para América Latina. RIL Editores.

Figueroa, F. (2020). Maristella Svampa: «Ser feminista y no ser ecologista es casi una contradicción» [Palabra Pública]. http://palabrapublica.uchile.cl/2020/11/03/maristella-svampa-ser-feminista-yno-ser-ecologista-es-casi-una-contradiccion/

Fuller, R. B. (1978). Operating manual for spaceship earth. Dutton.

Gatersleben, B., Murtagh, N., \& Abrahamse, W. (2014). Values, identity and proenvironmental behaviour. Contemporary Social Science, 9(4), 374-392.

Höhler, S. (2015). Spaceship Earth in the environmental age, 1960-1990. Pickering \& Chatto.

Kant, I. (2008). La metafísica de las costumbres. Tecnos.

Lindsay, G. (2021). Models of the Mind: How Physics, Engineering and Mathematics Have Shaped Our Understanding of the Brain. Bloomsbury Publishing.

Loncon Antileo, E. (2017). El Mapuzugun desde el Pensamiento Mapuche: Pasado, presente y futuro. Americanía: Revista de Estudios Latinoamericanos, 204-219.

Meadows, D., \& Club of Rome (Eds.). (1972). The Limits to growth: A report for the Club of Rome's project on the predicament of mankind. Universe Books.

Ministerio de Relaciones Exteriores de Bolivia (2010). Vivir Bien: mensajes y documentos sobre el Vivir Bien 1995-2010. Ministerio de Relaciones Exteriores.

Naess, A. (1992). Ecology, community and lifestyle: Outline of an ecosophy. Cambridge University Press.

Nast, C. (2019). What Is Eco-Fascism, the Ideology Behind Attacks in El Paso and Christchurch? GQ. https://www.gq.com/story/what-is-eco-fascism

Pereira da Silva, F. (2020). Sumak Kawsay-Suma Qamaña (Como conceito em construção para pensar assuntos internacionais e mundiais). En E. Devés Valdés \& S. T. Âlvarez (Eds.), Problemáticas internacionales y mundiales desde el pensamiento latinoamericano: Teorías, escuelas, conceptos, doctrinas, figuras (Primera edición, pp. 184-187). Ariadna Ediciones.

Protopapadakis, E. D. (2014). Environmental Ethics and Linkola's Ecofascism: An Ethics Beyond Humanism. Frontiers of Philosophy in China, 9(4), 586-601.

Ragusa, A., Svelato, A., Santacroce, C., Catalano, P., Notarstefano, V., Carnevali, O., Papa, F., Rongioletti, M. C. A., Baiocco, F., Draghi, S., D’Amore, E., Rinaldo, D., Matta, M., \& Giorgini, E. (2021). Plasticenta: First evidence of microplastics in human placenta. Environment International, 146, 106274. https://doi.org/10.1016/j.envint.2020.106274

Selcer, P. (2018). The postwar origins of the global environment: How the United Nations built Spaceship Earth. Columbia University Press. 
Snyder, T. (2018). The road to unfreedom: Russia, Europe, America. Tim Duggan Books.

Stern, A. M. (2019). White nationalists' extreme solution to the coming environmental apocalypse. The Conversation. http://theconversation.com/white-nationalistsextreme-solution-to-the-coming-environmental-apocalypse-121532

Stern, P. C. (2000). New Environmental Theories: Toward a Coherent Theory of Environmentally Significant Behavior. Journal of Social Issues, 56(3), 407-424. https://doi.org/10.1111/0022-4537.00175

Sperber, D., \& Hirschfeld, L. (1999). Culture, cognition, and evolution. En Robert A. Wilson \& Frank C. Keil (Eds.) MIT encyclopedia of the cognitive sciences, cxicxxxii. The MIT Press.

Svampa, M. (2015). Feminismos del Sur y ecofeminismo. Nueva Sociedad, 256, 127131.

van Boeckel, J. (1995). Interview with Arne Naess [The Call of the Mountain (Documentary)]. http://www.naturearteducation.org/R/Interviews/Naess4.htm

Ward, B. (1966). Spaceship earth: Earth science. Houghton Mifflin Co.

Zimmerman, M. E. (1995). The Threat of Ecofascism. Social Theory and Practice, 21(2), 207-238. https://doi.org/10.5840/soctheorpract199521210 